\title{
Capabilities of acoustic methods in testing of interference-fit joints
}

\author{
Anna Ryzhova ${ }^{1, *}$, Sergey Bekher ${ }^{1}$, and Aleksey Bobrov ${ }^{1}$ \\ ${ }^{1}$ Siberian Transport University, 630049 Novosibirsk, Russia
}

\begin{abstract}
Tightness of joints on railway wheelsets is controlled by instruments based on a shrink fit method. To date, the shrink fit method has been replaced by an interference fit method. As a result, standard values of tightness have doubled. Therefore, there is an urgent need to upgrade and develop new methods for testing interference-fit joints. A promising solution is to use acoustic methods based on influence of material properties on propagation patterns of elastic waves. The purpose of this study is to evaluate the possibility to test fixed detachable joints by acoustic methods. Reference samples of interference-fit joints with known interference fits $(29,72$ and $126 \mu \mathrm{m})$ were designed and produced. Experimental tests of samples and wheelset axles with inner bearing rings were conducted by a pulse-echo method and a method of free oscillations. The authors developed a procedure for determining the equivalent thickness of oil clearance using the proportionality factor of echo amplitude and sequence number of echo signals. When testing reference samples and loose bearing rings, frequency responses of the signals were obtained.
\end{abstract}

\section{Introduction}

Wheelsets with axle assemblies are the most important elements of undercarriage in railway cars. The wheelset manufacturing technology implies using fixed detachable joints made by the interference-fit method. Due to insufficient strength of these joints, which is partly associated with a loose inner bearing ring, axle assemblies of wheetsets may fail during operation.

At present, the interference fit of the inner bearing ring onto an axle journal is determined before installation of the axle assembly. Special devices are used to measure diameters of seating faces of the ring and axle in loose state. Based on a difference in diameters, the interference fit is estimated. However, measurement errors, scuffing from press fitting, and rotation of the ring during operation can deteriorate the quality of fit. In order to ensure traffic safety, reduce downtime of trains and cut costs of unscheduled repairs, these joints should be subject to complete inspection.

Acoustic methods described in [1-6] are the most advanced approach to testing interference fits. The ultrasonic method is based on a dependence of the reflection coefficient of ultrasonic waves at the joint interface on the tightness of fit [7]. The method

\footnotetext{
* Corresponding author: annalodo@yandex.ru
} 
of free oscillations is aimed at exposing a test part to a mechanical shock, and then measuring self-frequencies and the number of oscillation cycles over a time interval with a special instrument. Based on measured parameters, a mean value of interference fit is determined. The method was experimentally tested in [8] for testing joints of bronze bushings with cylindrical bodies with a diameter of $70 \mathrm{~mm}$ and bushings with articulated connecting rods with an interference fit of 30 to $120 \mu \mathrm{m}$. The amplitude of recorded waves is significantly affected by deviations between shapes of real and nominal surfaces, defects in test joints, and variations in surface roughness of joined parts. Therefore, the techniques need to be refined and verified for each specific type of joints.

The purpose of the paper is to study how the interference fit of an inner bearing ring onto an axle journal influences propagation patterns of acoustic waves. Unlike conventional methods for testing interference-fit joints, the proposed approach is based on a combination of active and passive acoustic methods. In this approach, the pulse-echo method is used for the local control of the tightness of fit, while the method of free oscillations is responsible for the integrated control of the entire joint. A dependence of informative parameters of diagnostic signals on interference fit was determined on the basis of reference samples. Influence of interfering factors was determined on the basis of real parts.

\section{Research methods}

The capabilities of the echo-pulse method (Fig. 1a) and the method of free oscillations (Fig. 1b) for testing interference-fit joints were experimentally studied on samples with a known interference fit [9] and on real parts.
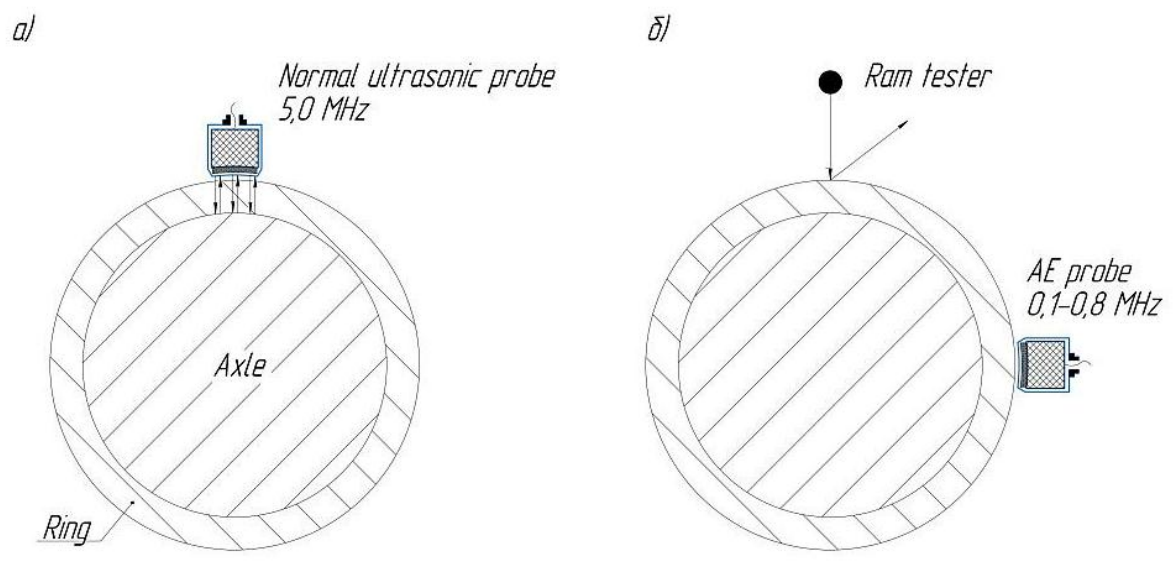

Fig. 1.Test diagrams for fixed detachable joints between a ring and an axle: a — pulse-echo method, $\mathrm{b}-$ method of free oscillations.

In case of the ultrasonic testing method, the thickness of a clearance between the ring and the shaft is used as an informative parameter. It determines acoustic properties of the interface between two media and represents the tightness of the fit of joined parts. For active control of interference-fit joints, a straight-beam single element transducer with an operating frequency of $5.0 \mathrm{MHz}$ was used to generate and receive ultrasonic waves. The transducers were connected to a USD-50 multipurpose pulse-echo flaw detector (State Register No. 52657-13). The ring -axle joint was scanned radially from the running surface of the bearing ring (Fig. 1a). A series of echo pulses re-reflected from the inner and outer cylindrical surfaces of bearing rings were recorded. $A_{1}, A_{2}, . ., A_{n}$ (Fig. 2). The amplitude of 
the $n^{\text {th }}$ pulse is satisfactorily described by a linear relationship with the proportionality factor $\alpha$ :

$$
A_{n}=\alpha \cdot n
$$

where $\alpha$ is the proportionality factor, which is determined by the attenuation coefficient, thickness of the ring, coefficients of reflection from the entry surface and interface between two media, as well as by a clearance between the ring and the shaft, $\mathrm{dB} ; n$ is the number of echo pulse.

The experimentally determined proportionality factor $\alpha$ makes it possible to estimate the thickness of the contact layer of the joint using the known dependencies [10-12]. Other things being equal, the thickness of the layer characterizes the mechanical stress in joined parts:

$$
\begin{gathered}
\operatorname{Lg}\left(R_{g}\right)=\frac{\alpha_{\Delta}-\alpha_{0}}{20}, \\
R_{g}=\frac{\left(\frac{z_{1}}{z_{2}}-\frac{z_{2}}{z_{1}}\right)^{2}}{4 \operatorname{ctg}\left(\frac{2 \cdot \pi \cdot h}{\lambda_{2}}\right)+\left(\frac{z_{1}}{z_{2}}+\frac{z_{2}}{z_{1}}\right)^{2}}
\end{gathered}
$$

where $R_{g}$ is the coefficient of reflection from the ring-clearance-shaft interface; $\alpha_{\Delta}$ and $\alpha_{0}$ are the proportionality factors for the reference sample with interference fit $\Delta$ and for the loose ring, respectively; $z_{1}$ is the acoustic impedance of the ring material (steel), which is equal to $46 \mathrm{MPa} \cdot \mathrm{s} / \mathrm{m} ; z_{2}$ is the acoustic impedance of the clearance medium (oil), which is equal to $1.3 \mathrm{MPa} \cdot \mathrm{s} / \mathrm{m} ; \mathrm{h}$ is the thickness of the clearance, $\mathrm{m} ; \lambda_{2}$ is the length of the acoustic wave in the second medium, $\mathrm{m}$.

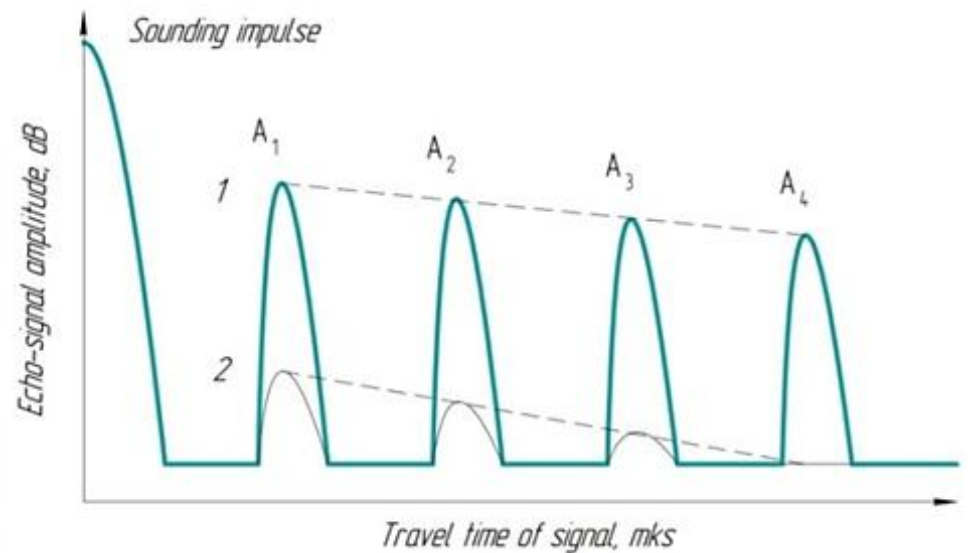

Fig. 2. A-scan with a series of re-reflected pulses: 1 - loose rings, 2 - sample with an interference fit of $72 \mu \mathrm{m}$.

In experiments with the passive control method, oscillations were excited by shock action with steel balls weighing $9 \mathrm{~g}$, freely falling from a height of $0.2-0.4 \mathrm{~m}$ inside a guide tube. The resulting oscillations were recorded with a GT-300 acoustic emission transducer. Signals were digitized by a Tektronix TDS 2014 oscilloscope and processed with specialpurpose software. The spectral composition of the signals and its response to changes in the interference fit were analyzed. 


\section{Experimental data and results}

Figure 2 shows a typical A-scan of an ultrasonic flaw detector with a series of re-reflected pulses recorded in a loose ring and a sample with an interference fit of $72 \mu \mathrm{m}$. The logarithm of the signal amplitude depends linearly on the pulse number with a correlation coefficient of more than 0.98 . Proportionality factors for different values of interference fit were calculated by the least-squares method (Fig. 3). The minimum slope $(1.9 \mathrm{~dB})$ of the characteristic curve is observed for the loose bearing ring. The maximum slope $(6.3 \mathrm{~dB})$ is observed for the reference bearing sample with an interference fit of $126 \mu \mathrm{m}$. For samples with a wide range of interference fits from 29 to $126 \mu \mathrm{m}$, the proportionality factors varies slightly — from 5.3 to $6.3 \mathrm{~dB}$.

The proportionality factors obtained at a repair depot do not correspond to the data determined with the samples. The difference can be attributed to the state of the seating face, which distorts the relationship of the proportionality factor for the amplitude of rereflected pulses and the sequence number with interference fit.

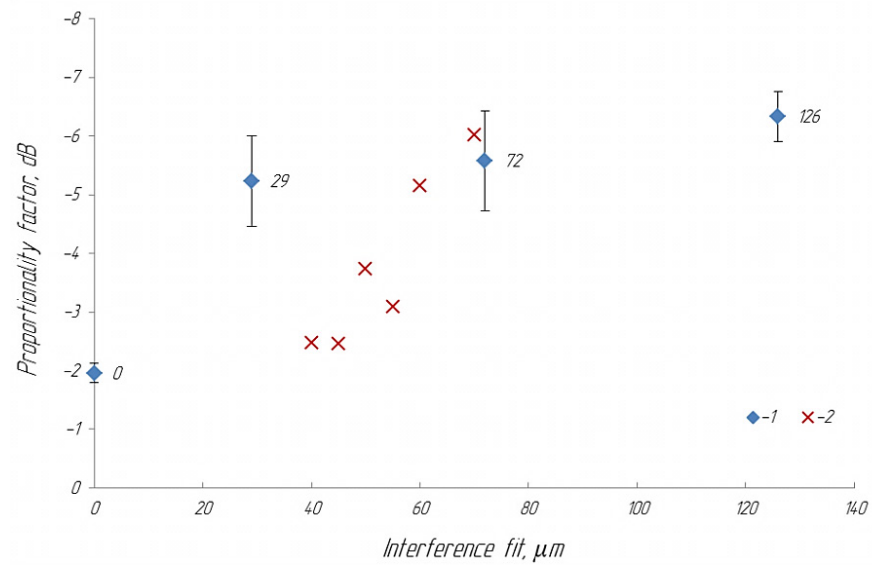

Fig. 3. Dependence of the proportionality factor on the interference fit: 1 - reference samples, $2-$ real parts.

Spectral compositions of the signals recorded by the self-oscillation method on the loose ring and the reference sample significantly differ from each other (Fig. 4). The frequency response in interference-fit joints does not contain low-frequency components with a frequency of less than $15 \mathrm{kHz}$. The spectral lines in the region from 15 to $33 \mathrm{kHz}$ also differ substantially. The width of the spectral lines measured at half-height is $0.1 \mathrm{kHz}$.

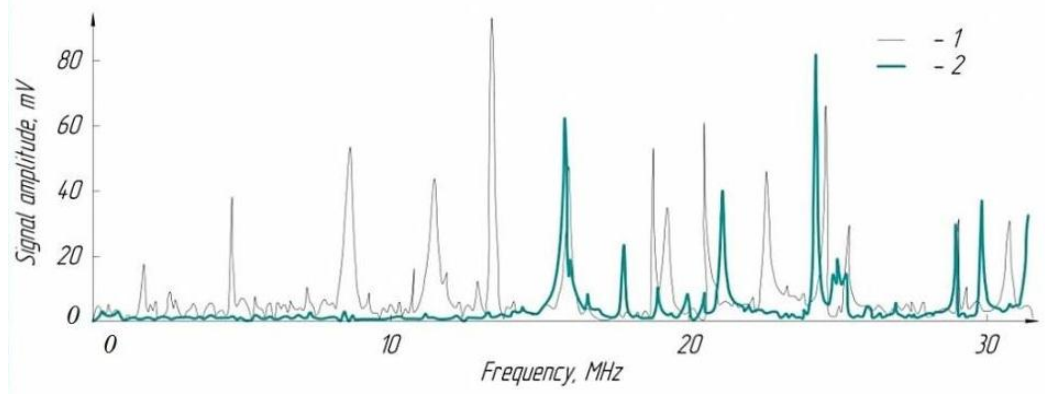

Fig. 4. Frequency responses of the signals received by an acoustic sensor for 1 - loose ring, 2 sample with an interference fit of $72 \mu \mathrm{m}$. 
The quality assessment of the joint by the passive method can be performed only if the position of the spectral lines changes with change in the interference fit. This is due to changes in acoustic and geometric parameters of the ring mounted onto the axle, as well as the transparency of the axle- ring interface. In turn, these changes influence the frequency response of free oscillations arising from the impact [13]. Figure 5 shows the frequency responses of signals recorded on the ring mounted on the axle with an interference fit of 29 and $72 \mu \mathrm{m}$. The most significant shift of the spectral lines occurs in the high-frequency region ( $24 \mathrm{kHz}$ or more), which can be used to estimate the interference fit.

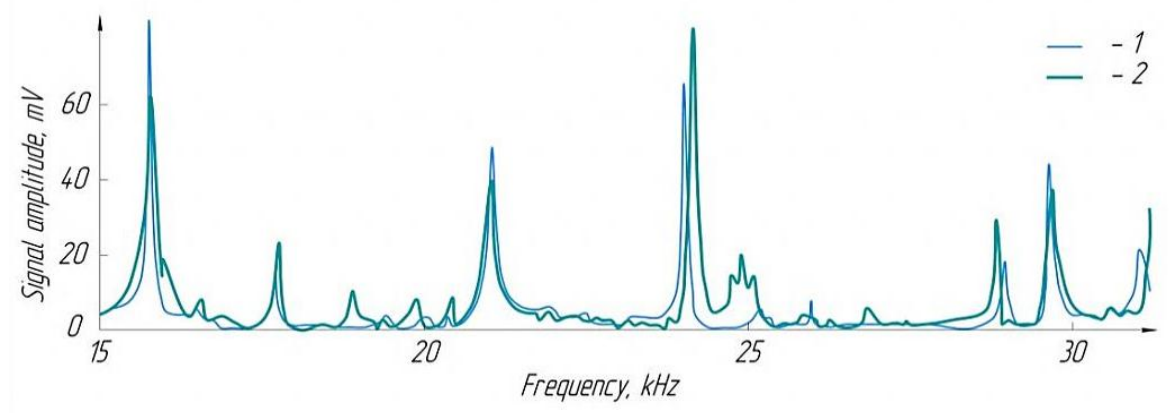

Fig. 5. Frequency responses of the signals received by an acoustic sensor for 1 - sample with an interference fit of $29 \mu \mathrm{m}, 2$ - sample with an interference fit of $72 \mu \mathrm{m}$.

\section{Conclusion}

The studied passive and active techniques aimed at controlling the tightness of interference fit of bearing rings onto an axle journal can be used to detect joints with a low interference fit of $29 \mu \mathrm{m}$ or less. The active pulse-echo method is based on indirect estimates of the thickness of contact layer using measured amplitudes of echo signals reflected from the joint interface. Using the reference samples with an interference fit of 29, 72 and $126 \mu \mathrm{m}$, the authors experimentally determined proportionality factors for amplitudes of echo pulses with their ordinal numbers 5.23, 5.58 and $6.34 \mathrm{~dB}$. The calculated thicknesses of the oil contact layer in the joint are $0.31,0.27$ and $0.22 \mathrm{~mm}$. In the conditions of a car repair depot, the deviation of the axle surface from the cylindrical shape has a significant effect on the thickness of the clearance and, therefore, on acoustic parameters of the joint. Consequently, the ultrasonic method is sensitive not only to interference fit, but also to out-of-roundness, conicity and surface roughness of parts.

In case of the passive method, the informative parameters for estimating the interference fit are self-frequencies in the range of 15 to $30 \mathrm{kHz}$. The characteristic width of the peaks measured at half-height is $0.1 \mathrm{kHz}$, which corresponds to the spectral shift with a change in the interference fit from 29 and $72 \mu \mathrm{m}$. The considered methods make it possible to detect joints with low interference fit. In order to increase the reliability of control procedures, further studies should be aimed at reducing the influence of interfering factors on the accuracy of interference fit control, through the use of a multifrequency pulse-echo method or optimization of the impact technique in the method of free oscillations.

\section{References}

1. V.V. Muravyev, V.A. Strizhak, L.V. Volkova, A.V. Pryakhin, Lokomotiv [Locomotive], 5, 39-41(2014). (in Russian)

2. I. Solodov, J. Bai, S. Bekgulyan, G. Busse, Appl. Phys. Lett, 99, 211911 (2011). 
3. L.N. Stepanova, E.S. Tenitilov, S.A. Bekher, Defektoskopija [Russian Journal of Nondestructive Testing], 9, 49-55 (2009). (in Russian)

4. A.G. Belonogov, S.A. Rodionov, A.M. Shanaurin, Defektoskopija [Russian Journal of Nondestructive Testing], 7, 90-92 (2004). (in Russian)

5. K.-Y. Jhang, International Journal of Precision Engineering and Manufacturing, Vol. 10 (1), 123-135 (2009).

6. I.I. Kozyatnik, T.V. Muravyev, Siberian Transport University Bulletin, 12, 73-81 (2005). (in Russian)

7. V.P. Ivannikov, A.V. Kabakova, Industrial laboratory. Diagnostics of materials, 81(9), 49-53 (2015). (in Russian)

8. B.A. Glagovskiy, I.B. Moskovenko, Nizkochastotnye akusticheskie metody kontrolja v mashinostroenii [Low-frequency acoustic methods of control in engineering] (Mashinostroenie, Leningrad, 1977). (in Russian)

9. A.O. Ryzhova, S.A. Bekher, A.L. Bobrov, "The acoustic waves propagation laws in the force-fit connections for test of the interference fit", JPCS (to be published).

10. V.V. Muravyev, O.V. Muravyeva, A.V. Platunov, Akusticheskaja tenzometrija $i$ strukturoskopija tonkih stal'nyh provolok [Acoustic tensometry and structuroscopy of thin steel wires] (ISTU Publishing House, Izhevsk, 2016) (in Russian).

11. V.A. Troitskiy, V.Yu. Popov, Ul'trazvukovoj kontrol' svarnyh soedinenij [Ultrasonic inspection of welded joints] (Feniks, Kazan, 2010) (in Russian).

12. L.M. Brekhovskikh, Volny v sloistyh sredah [Waves in layered medium] (Nauka, Moscow, 1973). (in Russian)

13. Yu.V. Lange, Akusticheskie nizkochastotnye metody i sredstva nerazrushajushhego kontrolja mnogoslojnyh konstrukcij [Acoustic low-frequency methods and nondestructive inspection aids of multiply construction] (Mashinostroenie, Moscow, 1991) (in Russian). 